León, M.P.; González-Martí, I. y Contreras, O.R. (2021) Development and Validation of the Preschoolers Body Scale. Revista Internacional de Medicina y Ciencias de la Actividad Física y $\begin{array}{llllll}\text { el Deporte } & \text { vol. } & 21 & \text { (84) } & \text { pp. } & 623-637 .\end{array}$ Http://cdeporte.rediris.es/revista/revista83/artdesarrollo1275.htm

DOI: https://doi.org/10.15366/rimcafd2021.83.013

\title{
ORIGINAL
}

\section{DESARROLLO Y VALIDACIÓN DE LA ESCALA CORPORAL PARA PREESCOLARES}

\section{DEVELOPMENT AND VALIDATION OF THE PRESCHOOLERS BODY SCALE}

\author{
León, M.P.'; González-Martí, I. ${ }^{2}$ y Contreras, O.R. ${ }^{3}$ \\ 1 Investigadora predoctoral en formación FPU. Departamento de Didáctica de la Expresión \\ Musical, Plástica y Corporal. Facultad de Educación de Albacete. Universidad de Castilla-La \\ Mancha (España) MariaPilar.Leon@uclm.es \\ ${ }^{2}$ Profesora Contratada Doctora. Departamento de Didáctica de la Expresión Musical, Plástica y \\ Corporal. Facultad de Educación de Cuenca. Universidad de Castilla-La Mancha (España) \\ Irene.GMarti@uclm.es \\ ${ }^{3}$ Catedrático de Universidad. Departamento de Didáctica de la Expresión Musical, Plástica y \\ Corporal. Facultad de Educación de Albacete. Universidad de Castilla-La Mancha (España) \\ Onofre.CJordan@uclm.es
}

Agradecimientos I Acknowledgements: Este estudio fue financiado por los subprogramas de Formación y de Movilidad incluidos en el Programa Estatal de Promoción del Talento y su Empleabilidad, en el marco del Plan Estatal de Investigación Científica y Técnica y de Innovación 2013-2016.

Código UNESCO I UNESCO code: 6302.02 Psicología social / Social psychology

Clasificación del Consejo de Europa / Council of Europe classification: 17. Otras: / Others: Imagen corporal / Body image

Recibido 23 de julio de 2019 Received July 2019, 23

Aceptado 15 de septiembre de 2019 Accepted September 15, 2019

\section{RESUMEN}

El objetivo del estudio fue desarrollar y validar un instrumento para medir la percepción del tamaño corporal y la insatisfacción corporal en preescolares. La Escala Corporal para Preescolares (PBS) estuvo compuesta por cuatro figuras corporales (versiones masculina y femenina) en posición frontal y lateral, representando cuatro categorías de peso. La escala fue administrada a 244 preescolares de 4 a 6,4 años, de los cuales 42 participaron en el estudio piloto y 202 en la fiabilidad del instrumento, la cual se reforzó con un proceso de validación y fiabilidad con 10 pediatras expertos de entre 50 y 65 años. Los 
resultados revelaron una buena validez y una fiabilidad de moderada a alta, que fue mejorando conforme los niños fueron mayores, especialmente con las figuras de perfil. Se comprobó que PBS es un instrumento adecuado para evaluar la percepción corporal y la insatisfacción corporal, particularmente entre participantes mayores de 5 años.

PALABRAS CLAVE: preescolares, imagen corporal, percepción corporal, insatisfacción corporal, validez, fiabilidad, instrumento

\section{ABSTRACT}

The aim was to develop and validate an instrument to measure the body size perception and body dissatisfaction among preschoolers. The Preschoolers Body Scale (PBS) is composed of two sets of four figures (male and female versions) in front and profile views, representing four weight categories. The scale was administered to a total of 244 preschoolers aged 4 to 6.4 years, 42 participated in the pilot study and 202 took part in the instrument's reliability study. Also, 10 expert pediatricians aged 50 to 65 years participated in the validation and reliability process. Results revealed good validity and moderateto-high reliability, which improved as children became older. We conclude that the PBS is a reliable and appropriate instrument to measure body perception in preschoolers, particularly among participants older than 5 years.

KEYWORDS: preschoolers, body image, body size percepction, body dissatisfaction, validity, reliability, instrument.

\section{INTRODUCCIÓN}

En el marco de la imagen corporal, los componentes perceptivo (i.e., estimación de las dimensiones del cuerpo) y cognitivo-afectivo (i.e., sentimientos, actitudes, emociones y valoraciones del cuerpo) (Thompson, 1990) han sido ampliamente estudiados, especialmente entre preadolescentes y adolescentes, dado que se les considera poblaciones de riesgo para sufrir insatisfacción corporal y trastornos de la conducta alimentaria (Gómez-Mármol, Sánchez-Alcaraz, MolinaSaorín y Bazaco, 2017; Rodgers, Paxton y McLean, 2014). Sin embargo, la literatura teórica y empírica indica que el desarrollo de la imagen corporal y el comienzo de la preocupación con el cuerpo aparece en edades tempranas (Smolak, 2012), donde existe una escasez de investigación (Lombardo, Battagliese, Pezzuti y Lucidi, 2014).

Hasta el momento, estudios previos revelan que la mayoría de los preescolares son inexactos en la percepción de su tamaño corporal (Ambrosi-Randic y Tokuda, 2004; Cramer y Steinwert, 1998; Meers, Koball, Wagner, Laurene y Musher-Eizenman, 2011; Ra, Yun y Cho, 2016), especialmente el género femenino (Ambrosi-Randic, 2000; Holub, 2008) y los niños con exceso de peso (Cramer y Steinwert, 1998; Burgess y Broome, 2012; Tremblay, Lovsin, Zecevic y Larivière, 2011). Teniendo en cuenta estos resultados, se ha sugerido que la 
exactitud en la percepción corporal podría desarrollarse en edades más avanzadas (Ambrosi-Randic y Tokuda, 2004).

Esta percepción de sí mismo es complementada con una dimensión valorativa y afectiva del yo, es decir, en qué medida el niño valora sus características y competencias, cómo de satisfecho o insatisfecho, de contento o descontento se siente respecto a cómo es (Palacios, Marchesi y Colll, 1990). En relación a dicha dimensión, algunos estudios previos han demostrado que un gran porcentaje de niños revelan insatisfacción corporal (Ambrosi-Randic y Tokuda, 2004; Dohnt y Tiggemann, 2006a, 2006b; Li, Hu, Ma, Wu y Ma, 2005; Musher-Eizenman, Holub, Edwards-Leeper, Persson y Goldstein, 2003; Pallan, Hiam, Duda y Adab, 2011; Ra et al., 2016; Tremblay et al., 2011, Wong et al., 2013), mientras que otros han encontrado que la mayoría de niños estaban contentos con su cuerpo (Burgess y Broome, 2012; Damiano et al., 2015; Davison, Markey y Birch, 2000; Xu y Nerren, 2008), demostrando más preocupación con su pelo y ropa que con su tamaño o forma corporal (Hayes y Tantleff-Dunn, 2010; McCabe et al., 2007). Por tanto, los resultados son variados y poco concluyentes.

Durante la etapa de preescolar, la percepción del tamaño corporal y la insatisfacción corporal suelen medirse con instrumentos visuales de aplicación fácil y rápida (Gardner, Jappe y Gardner, 2009; Truby y Paxton, 2002), tal y como recomiendan algunos investigadores (Collins, 1991; Tremblay et al., 2011), ya que los niños tan jóvenes pueden tener limitaciones para verbalizar sus ideas y describir sus representaciones. Los instrumentos visuales más utilizados son las escalas de figuras o siluetas corporales (Gardner et al., 2009; Hill, 2012), compuestas por cuerpos de diferentes tamaños ordenados de más delgado a más obeso (Collins, 1991; Lerner y Gellert, 1969; Stunkard, Sorensen y Schulsinger, 1983; Tiggemann y Pennington, 1990; Truby y Paxton, 2002). Estas escalas normalmente contienen siete o nueve siluetas o figuras con rostros y alturas idénticas, de modo que la única dimensión que varía es el tamaño del cuerpo. Para evaluar la percepción corporal se pregunta a los niños qué figura se parece más a ellos, obteniéndose una puntuación de discrepancia que compara el Índice de Masa Corporal (IMC) del niño con el IMC de la figura seleccionada como actual, mientras que para la insatisfacción corporal se obtiene otra puntuación de discrepancia restando la figura que los niños seleccionan como percibida a la que les gustaría tener; es decir, la figura deseada (Collins, 1991; Truby y Paxton, 2002).

Diversos estudios han señalado que una de las causas de la percepción inexacta del tamaño corporal podría deberse a la inexactitud de los instrumentos de evaluación (Collins, 1991; Holub, 2008; Pallan et al., 2011) ya que, al no existir instrumentos validados para población infantil, se han utilizado escalas diseñadas para poblaciones de mayor edad, lo cual pone en entredicho su adecuación. En algunos casos, se han realizado adaptaciones de las escalas para facilitar la tarea a los preescolares, reduciendo por ejemplo el número de figuras (Meers et al., 2011). La inexactitud de las escalas corporales también ha sido criticada en tanto que algunas se componen de dibujos subjetivos que no se corresponden con cuerpos reales, lo cual dificulta una medición adecuada o exacta del componente perceptivo de la imagen corporal (Gardner, 2012; Lombardo et al., 2014). 
Con el fin de superar las limitaciones expuestas, el objetivo del estudio fue desarrollar y validar un instrumento basado en figuras corporales reales creadas a partir de fotografías de niños para medir la percepción del tamaño corporal y la insatisfacción corporal en preescolares. De acuerdo con este objetivo, se formuló la hipótesis de que el instrumento demostraría una validez y fiabilidad adecuadas, siendo más fiable con los niños de mayor edad.

\section{MATERIAL Y MÉTODO}

\subsection{Participantes}

Un total de 244 preescolares formaron parte del estudio. De ellos, un subgrupo de 42 niños caucásicos de 4,4 a 6,3 años (22 niñas, 20 niños, Medad $=5,25 \pm$ $0,54)$ participó en el estudio piloto, y los 202 niños restantes de 4 a 6,4 años (97 niñas, 105 niños, $M_{\text {edad }}=5,24 \pm 0,63$ ) lo hicieron en el proceso de fiabilidad del instrumento. Todos los participantes fueron seleccionados de 13 colegios de Albacete, España. La etnia de los niños fue reportada por los padres, siendo el $98 \%$ caucásicos, el $1 \%$ Americanos y el $1 \%$ restante, Negros. Los niños fueron divididos en cinco grupos según su edad de la siguiente manera: 4 a 4,4 años $\left(n_{+}=10 ; n_{0}=19\right) ; 4,5$ a 4,9 años $\left(n_{+}=13 ; n_{0}=24\right) ; 5$ a 5,4 años $\left(n_{+}=22 ; n_{3}=\right.$ $22) ; 5,5$ a 5,9 años $\left(n_{+}=36 ; n_{0}=29\right) ;$ y 6 a 6,4 años $\left(n_{+}=16 ; n_{0}=11\right)$. Esta clasificación fue realizada debido a las diferencias cognitivas que existen entre los niños nacidos en diferentes meses del mismo año natural, lo cual se conoce como el efecto de la edad relativa (Barnsley, Thompson y Barnsley, 1985). Además de la fiabilidad obtenida con niños, se realizó un proceso de validación y fiabilidad con 10 pediatras, siete hombres y siete mujeres, de entre 50 y 65 años $\left(M_{\text {edad }}=58,7 \pm 5,83\right)$; todos ellos con más de 10 años de experiencia en su profesión.

\subsection{Instrumentos}

\subsubsection{Escala Corporal para Preescolares (PBS).}

El instrumento utilizado fue diseñado a partir de fotografías y consta de dos escalas compuestas por cuatro figuras corporales reales pertenecientes a cuatro niños y cuatro niñas de 4 a 6 años. Una escala muestra las figuras en posición lateral y otra en posición frontal. La primera figura corporal corresponde a un niño con muy bajo peso (percentil $3-\mathrm{IMC}_{\text {niño }}=13,13$ e $\mathrm{IMC}_{\text {niña }}=13,03-$ ), la figura 2 representa un peso normal (percentil 50 -IMC $\mathrm{IMño}_{\text {nin }}=16$ e IMC $\left.\mathrm{IM}_{\text {niña }}=15,06-\right)$, la figura 3 pertenece a un niño con sobrepeso (percentil $85-\mathrm{IMC}_{\text {niño }}=17,1$ e $\mathrm{IMC}_{\text {niña }}=$ 17,06-) y finalmente, la figura 4 refleja un niño con obesidad (percentil >99 $I \mathrm{IC}_{\text {niño }}=21,03$ e IMC $\left.\mathrm{IMña}_{\text {nas }}=21,25-\right)$. Todas las figuras tienen la misma altura y están colocadas en orden de más delgada a más obesa.

Para evitar la tendencia a elegir la respuesta central (Matas, 2018), la escala estuvo formada por un número par de figuras. Además, debido a la temprana edad de los participantes, se eligió un número reducido de figuras, ya que como han sugerido Lombardo et al. (2014) y Paxton y Damiano (2017), los preescolares podrían tener dificultades para discriminar las pequeñas diferencias 
que existen entre las figuras adyacentes de una escala numerosa. Aunque se desconoce cuál es el número ideal de figuras, Gardner y Brown (2010) afirman que, a pesar de que en algunas escalas se utilizan muchas figuras, realmente sólo se hace uso de tres o cuatro en la mayoría de los casos.

Con el objetivo de diseñar el instrumento se obtuvo el peso y la talla de 61 sujetos. Tras ello, se calcularon sus IMC (peso $\mathrm{kg} /$ altura $\mathrm{m}^{2}$ ) y percentiles de IMC siguiendo los estándares de crecimiento infantil de la Organización Mundial de la Salud (OMS, 2006) y se agrupó a los niños en cuatro categorías de peso: muy bajo o bajo peso (percentil $\leq 10$ ), peso normal o normopeso (percentiles 15-75), sobrepeso (percentiles 85-95) y obesidad (percentil $\geq 97$ ). Finalmente, se seleccionó a un niño de cada sexo y categoría de peso para ser fotografiados de frente y perfil.

Las fotografías fueron tomadas individualmente por un investigador en presencia de los padres y en una sala separada y tranquila. Los niños, vestidos con ropa interior, se ubicaron de pie a 20 centímetros de la pared y 150 centímetros de la cámara y el trípode. Las cabezas de los niños no se fotografiaron para mantener su anonimato y facilitar que durante la evaluación de la percepción corporal éstos centrasen su atención en el cuerpo de las figuras, y no solo en los rasgos faciales. Además, tal como apuntan Gardner et al. (2009) la omisión de dichos rasgos es fundamental para que el instrumento sea apropiado para su administración con otros grupos étnicos, debido a que las escalas existentes reflejan la etnia caucásica. Un ejemplo de esta limitación puede verse en el estudio de Li et al. (2005), quienes adaptaron los rasgos faciales de la escala de Collins (1991) a su cultura.

Finalmente, las fotografías obtenidas se editaron con Adobe Photoshop CC 2015 para obtener la primera versión del instrumento, mostrándose un ejemplo de la escala femenina en la Figura 1.

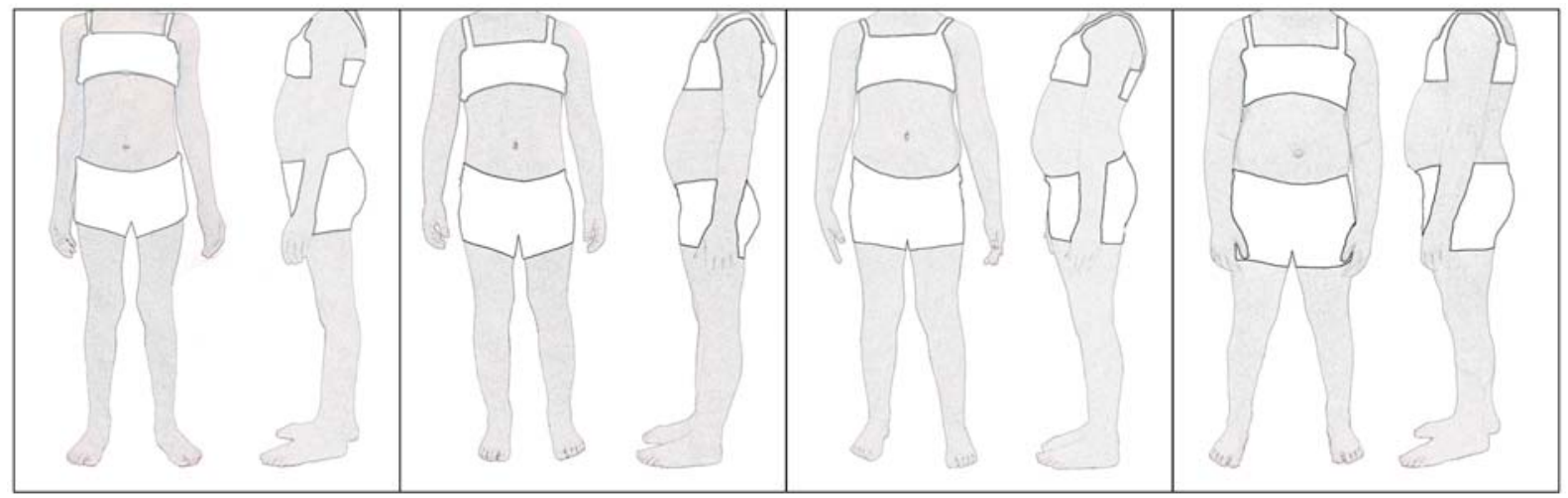

Figura 1. Primera versión femenina de PBS.

Utilizando la versión femenina y masculina del instrumento, se realizó un estudio piloto para comprobar su adecuación. Primeramente, la escala estuvo compuesta por cuatro cuadrados que contenían la figura de perfil y frente de cada niño fotografiado. Tras el estudio piloto, el instrumento fue modificado, dividiendo la escala en dos, una con las figuras de perfil y otra con las de frente. Esta modificación se realizó al comprobar que muchos niños, especialmente los de menor edad, revelaban dificultades para comprender que las dos figuras de 
un cuadrado pertenecían al mismo niño. La versión final del instrumento para el género masculino y femenino se ilustra en las Figuras 2 y 3.
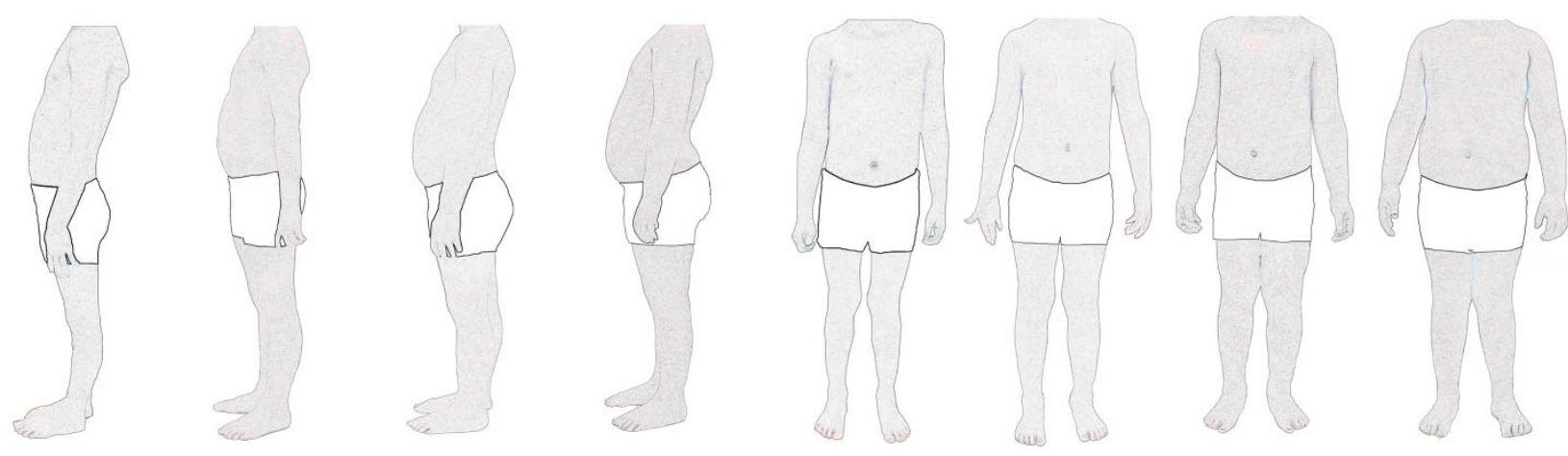

Figura 2. Versión final masculina de PBS.
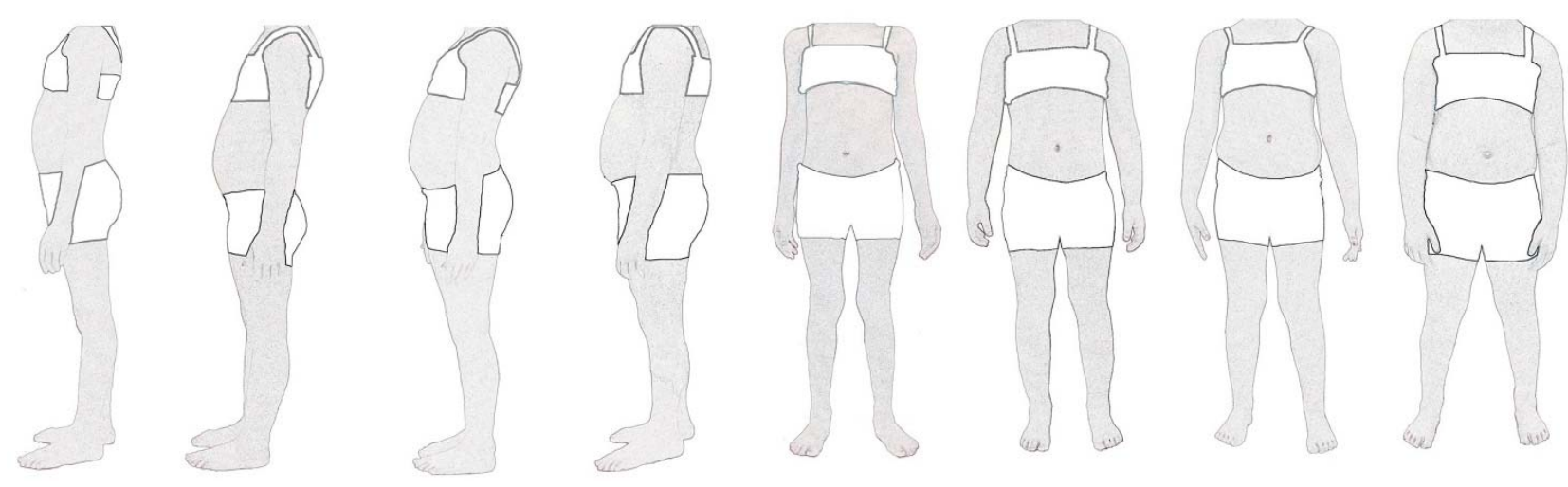

Figura 3. Versión final femenina de PBS.

A través de las escalas mostradas y con el propósito de medir la percepción del tamaño corporal y la insatisfacción corporal, se les pregunta a los niños que identifiquen su figura percibida (i.e., ¿Qué niño crees que se parece más a ti?) y su figura deseada (i.e., ¿A qué niño te gustaría parecerte?). Para conocer si la percepción de los niños es exacta, se calcula una puntuación de discrepancia (figura real - figura percibida), mientras que para conocer la insatisfacción corporal, se obtiene otra puntuación de discrepancia restando la figura deseada a la percibida. Ambas puntuaciones oscilarían entre 3 y -3 .

\subsection{Medidas}

\subsubsection{Antropometría}

Se midió y pesó a los niños para calcular su IMC, percentiles y puntaciones $z$ de IMC. La altura, medida con ropa ligera y sin zapatos, se obtuvo con un tallímetro portátil con una precisión de $0,1 \mathrm{~cm}$ (Tanita HR-001), y el peso se midió con una báscula digital (Tanita HD-366) con una precisión de 0,1 kg. Estas medidas fueron tomadas por un investigador especializado con certificación de antropometría de primer nivel expedido por la International Society for the Advancement of Kinanthropometry (ISAK). 


\subsubsection{Validez y fiabilidad}

Los niños participaron únicamente en la fiabilidad del instrumento, ya que la mayoría de los estudios revelan las dificultades de los preescolares para percibir su tamaño corporal, lo cual podría sesgar la validez del instrumento. Siguiendo el procedimiento de estudios previos (Collins, 1991; Lerner y Gellert, 1969; Pallan et al., 2011), se utilizó un diseño test-retest de 72 horas, preguntando verbalmente a los niños sobre su figura percibida y deseada.

La fiabilidad del instrumento obtenida con los niños fue reforzada con un proceso de validez y fiabilidad con 10 pediatras expertos, como también hicieron Wells, Goldstein y Bentley (2018). El número de expertos fue determinado siguiendo las recomendaciones de Landeta (2002), quien propone un mínimo de siete para obtener un juicio o valoración sobre un tema.

La validez de constructo con los expertos se midió observando el grado de correspondencia entre el IMC de los niños de las fotografías y el de las figuras de la escala seleccionadas por los pediatras (Swami, Salem, Furnham y Tovée, 2008), mientras que la fiabilidad se calculó mediante el acuerdo interobservador con el fin de obtener el grado de concordancia entre las respuestas de los participantes (Fleiss, 1981).

\subsection{Procedimiento}

El presente estudio fue aprobado por el Comité de Ética de Investigación del Hospital de Albacete y por la Delegación Provincial de Educación de Castilla-La Mancha. Asimismo, se contó con la aprobación de los directores de los centros educativos. Dado que la muestra estuvo compuesta por niños, los padres dieron su consentimiento por escrito para participar en el estudio, mientras que los niños dieron su consentimiento verbal antes de la recogida de datos, que se llevó a cabo en horario escolar.

La antropometría de los niños se obtuvo en una sala aislada a través de la medición de altura y peso, siguiendo para ello el protocolo de la ISAK (2001). Para la altura, el sujeto estuvo situado parado con los pies juntos y las nalgas y parte superior de la espalda descansando sobre el tallímetro y ubicando la cabeza en el plano Frankfort; mientras que para la medición del peso, el sujeto se colocó en el centro de la báscula y con el peso distribuido equitativamente sobre ambos apoyos.

Una vez obtenidos los datos de antropometría, se tomaron algunas fotografías y se diseñó la escala anteriormente explicada. Tras ello, cada niño fue entrevistado muy brevemente por un investigador entrenado para conocer su figura percibida y deseada. Durante esta tarea, el participante estuvo sentado en una silla pequeña frente a una pantalla de 17 pulgadas, la cual se ubicó en una mesa a la altura de los ojos del niño y a una distancia de $30 \mathrm{~cm}$. Se desarrolló un protocolo para garantizar un procedimiento estandarizado que no influyese a los niños en sus respuestas. 
Las figuras, cuyo tamaño era de $18 \times 7 \mathrm{~cm}$, fueron proyectadas en la pantalla en orden ascendente, de menor a mayor percentil de IMC, y con un fondo blanco, mostrando primero la escala de figuras de perfil y posteriormente la escala de frente. Este proceso se repitió a los tres días para obtener la fiabilidad test-retest.

Por otro lado, el mismo investigador llevó a cabo la toma de datos con todos los pediatras, quienes dieron su consentimiento informado para participar. Se les mostraron aleatoriamente 14 fotografías correspondientes a siete niños y siete niñas de diferentes estados de peso. Cada una de estas fotografías, tanto de frente como de perfil, fue proyectada individualmente durante 30 segundos en una pantalla de 24 pulgadas, mientras que las escalas de figuras les fueron entregadas impresas en un documento de tamaño estandarizado. En una hoja de registro, los pediatras anotaron qué figura de la escala se relacionaba con cada fotografía. En ningún momento se indicó el estado de peso que representaba cada figura o fotografía para no condicionar o sesgar sus respuestas.

\subsection{Análisis estadístico}

El análisis fue realizado con SPSS Statistics 24.0, obteniendo primeramente los estadísticos descriptivos. Para hallar la fiabilidad test-retest con los niños se realizaron correlaciones de Spearman y pruebas de los rangos con signo de Wilcoxon, haciendo un análisis estratificado por sexo y edad. La validez de constructo con los pediatras se calculó mediante correlaciones de Spearman, mientras que la Kappa de Fleiss fue utilizada para la fiabilidad interobservador, dada la naturaleza de las variables y la existencia de más de dos observadores. Por otro lado, los datos antropométricos de los niños se calcularon con el software AnthroPlus 1.0.4 (OMS, 2007).

\section{RESULTADOS}

\subsection{Antropometría}

El IMC de los niños, excluyendo a aquellos del estudio piloto, varió entre 12,19 y $21,25(M=15,45 \pm 1,66)$. El $13,1 \%$ de los participantes tenía bajo peso, el $72,1 \%$ normopeso, $8,2 \%$ sobrepeso y $6,6 \%$ obesidad.

\subsection{Validez y fiabilidad}

Respecto a la validez de constructo, los resultados mostraron una correlación alta y significativa entre el IMC de los niños de las fotografías y el IMC de las figuras seleccionadas por los pediatras $(\rho=0,85, p<, 005)$. Además, la fiabilidad interobservador demostró una concordancia sustancial teniendo en cuenta todas las figuras de la escala, con una Kappa de Fleiss de 0,61 (Fleiss, 1981). Este coeficiente se considera moderado entre 0,41 y 0,6, mientras que a partir de 0,61 la concordancia es considerable. Analizando la fiabilidad por figura, los índices oscilan entre 0,49 y 0,81 , existiendo mayor concordancia entre los expertos con las fotografías de niños obesos y menor acuerdo con las de niños con 
normopeso. Para la figura con bajo peso, el grado de acuerdo fue de 0,55 , mientras que con la figura de sobrepeso fue de 0,58.

La fiabilidad test-retest de los niños fue calculada mediante correlaciones de Spearman, observando así la consistencia temporal entre test (T1) y retest (T2). Asimismo, se obtuvieron las medias y desviaciones típicas de las figuras elegidas como percibidas en el T1 y T2 y la prueba de los rangos con signo de Wilcoxon para comparar las medias entre los dos tiempos. Los resultados por edad y género se muestran en la Tabla 1.

Tabla 1. Fiabilidad test-restest para la figura percibida con la escala de perfil y frente, según

\begin{tabular}{|c|c|c|c|c|c|c|}
\hline \multirow[b]{2}{*}{ Grupos } & \multicolumn{2}{|c|}{$\begin{array}{c}\text { T1 Figura percibida. } \\
M(S D)\end{array}$} & \multicolumn{2}{|c|}{$\begin{array}{c}\text { T2 Figura percibida } \\
M(S D)\end{array}$} & \multicolumn{2}{|c|}{$\begin{array}{c}\text { Correlación T1-T2 } \\
\text { figura percibida }\end{array}$} \\
\hline & Perfil & Frente & Perfil & Frente & Perfil & Frente \\
\hline Todos $(N=202)$ & $1,68(1)$ & $2,05(1,01)$ & $1,68(0,97)$ & $1,91(0,98)$ &, $557^{* *}$ &, $408^{* *}$ \\
\hline Chicos $(n=105)$ & $1,68(1,07)$ & $2,14(1,04)$ & $1,66(1,01)$ & $2,02(1,09)$ &, $689^{* *}$ &, $404^{* *}$ \\
\hline Chicas $(n=97)$ & $1,68(0,91)$ & $1,95(0,97)$ & $1,71(0,92)$ & $1,79(0,85)$ &, $402^{* *}$ &, $396^{\star *}$ \\
\hline $\begin{array}{l}4-4,4 \text { años } \\
(n=29)\end{array}$ & $1,93(1,19)$ & $2,41(1,26)$ & $1,83(1,10)$ & $2,03(1,14)$ &, $387^{*}$ & ,216 \\
\hline $\begin{array}{l}4,5-4,9 \text { años } \\
(n=37)\end{array}$ & $2,08(1,22)$ & $1,89(0,96)$ & $2(1,13)$ & $2,05(1,07)$ &, $600^{* *}$ & ,029 \\
\hline $\begin{array}{l}5-5,4 \text { años } \\
(n=44)\end{array}$ & $1,45(0,79)$ & $2,16(0,93)$ & $1,52(0,97)$ & $1,82(0,92)^{*}$ &, $458^{* *}$ &, $439^{* *}$ \\
\hline $\begin{array}{l}5,5-6,4 \text { años } \\
(n=65)\end{array}$ & $1,51(0,80)$ & $1,98(0,91)$ & $1,59(0,79)$ & 1,88 $(0,85)$ &, $617^{* *}$ &, $592^{* *}$ \\
\hline $\begin{array}{l}6-6,4 \text { años } \\
(n=27)\end{array}$ & $1,63(1)$ & $1,85(1,06)$ & $1,56(0,93)$ & $1,81(1,11)$ &, $641^{* *}$ &, $718^{\star *}$ \\
\hline
\end{tabular}

$$
\begin{aligned}
& { }^{* *} p<.001 \\
& { }^{*} p<.005
\end{aligned}
$$

Atendiendo a la figura percibida, el coeficiente de correlación global para la fiabilidad test-retest fue de 0,55 con la escala de perfil y 0,40 con la de frente. Esta fiabilidad fue notablemente diferente según el género con las figuras de perfil, ya que los niños demostraron mayor fiabilidad que las niñas $(\rho=0,68, p<$ $, 001$ vs. $\rho=0,40, p<, 001)$.

Al realizar un análisis por edad, se observó un aumento en la fiabilidad. Siguiendo el criterio de Landis y Koch (2012), antes de los 4,4 años se observa baja o nula fiabilidad del instrumento, mientras que ésta fue moderada en los niños de 4,5 a 4,9 años con la escala de perfil $(\rho=0,60, p<, 001)$ y con ambas escalas entre 5 y 5,4 años. Sin embargo, es a partir de los 5,5 años cuando los niños revelaron mayor fiabilidad en ambas escalas (perfil: $\rho=0,61, p<, 001$ y frente: $\rho=0,59, p<, 001$ ), la cual aumentó a los 6 años (perfil: $\rho=0,64, p<, 001$ y frente: $\rho=0,71, p<, 001)$.

El test de rangos de Wilcoxon sólo reveló diferencias de medias significativas entre el T1 y T2 en el grupo de edad de 5 a 5,4 años $(Z=-2.060, p=, 039)$, ya que la puntuación media en el test fue de $1,52(0,97)$ y en el retest de $1,82(0,92)$. Teniendo en cuenta a todos los participantes, no hubo diferencias de medias con las figuras de perfil $(Z=-, 071, p=, 943)$ y de frente $(Z=-1.680, p=, 093)$, así como tampoco las hubo por género con las figuras de perfil (chicos: $Z=-, 460, p$ 
$=, 646$; chicas: $Z=-, 366, p=, 714)$ ni con las figuras de frente (chicos: $Z=$ $-1.099, p=, 272$; chicas: $Z=-1.323, p=, 186)$.

En cuanto a la figura ideal, la Tabla 2 refleja una fiabilidad más baja que con la figura percibida, ya que se obtuvo una correlación de 0,46 y 0,36 respectivamente con la escala de perfil y de frente. La fiabilidad test-retest mejoró a partir de los 5 años con ambas escalas, alcanzando las puntuaciones más altas a los 6 años (perfil: $\rho=0,73, p<, 001$ y frente: $\rho=0,50, p<, 001$ ). En cuanto al género, los chicos demostraron mayor fiabilidad que las chicas con ambas escalas [chicos (perfil: $\rho=0,52, p<, 001$ y frente: $\rho=0,39, p<, 001$ ) vs. chicas (perfil: $\rho=0,37, p<, 001$ y frente: $\rho=0,28, p<, 001)$ ].

Con la figura ideal, la prueba de rangos de Wilcoxon no arrojó ninguna diferencia significativa entre las medias del T1 y T2 (figuras de perfil: $Z=-1.467, p=, 142$; figuras de frente: $Z=-0,136, p=, 892)$.

Tabla 2. Fiabilidad test-restest para la figura ideal con la escala de perfil y frente, según género

\begin{tabular}{|c|c|c|c|c|c|c|}
\hline \multirow[b]{2}{*}{ Grupos } & \multicolumn{2}{|c|}{$\begin{array}{c}\text { T1 Figura ideal } \\
M(S D)\end{array}$} & \multicolumn{2}{|c|}{$\begin{array}{c}\text { T2 Figura ideal } \\
M(S D)\end{array}$} & \multicolumn{2}{|c|}{$\begin{array}{l}\text { Correlación T1- } \\
\text { T2 figura ideal }\end{array}$} \\
\hline & Perfil & Frente & Perfil & Frente & Perfil & Frente \\
\hline Todos $(N=202)$ & $1,82(1,01)$ & $2(0,97)$ & $1,72(0,96)$ & $2(1,01)$ &, $461^{* *}$ &, $365^{* *}$ \\
\hline Chicos $(n=105)$ & $1,85(1,09)$ & $2,15(1)$ & $1,71(1,05)$ & $2,14(1,12)$ &, $527^{* *}$ &, $395^{* *}$ \\
\hline Chicas $(n=97)$ & $1,79(0,92)$ & $1,84(0,90)$ & $1,72(0,85)$ & $1,85(0,87)$ &, $377^{* *}$ &, $287^{* *}$ \\
\hline $\begin{array}{l}4-4,4 \text { años } \\
(n=29)\end{array}$ & $2,10(1,20)$ & $2,34(1,17)$ & $1,97(1,11)$ & $2,48(1,05)$ & ,240 & ,352 \\
\hline $\begin{array}{l}4,5-4,9 \text { años } \\
(n=37)\end{array}$ & $2,39(1,20)$ & $2,05(0,97)$ & $2,14(1,20)$ & $2,14(1,13)$ &, $495^{\star *}$ &,- 032 \\
\hline $\begin{array}{l}5-5,4 \text { años } \\
(n=44)\end{array}$ & $1,75(0,86)$ & $2,18(1,04)$ & $1,73(0,89)$ & $2,05(1,09)$ &, $347^{*}$ &, $375^{*}$ \\
\hline $\begin{array}{l}5,5-6,4 \text { años } \\
(n=65)\end{array}$ & $1,54(0,84)$ & $1,89(0,83)$ & $1,42(0,73)$ & $1,85(0,83)$ &, $341^{* *}$ &, $456^{* *}$ \\
\hline $\begin{array}{l}6-6,4 \text { años } \\
(n=27)\end{array}$ & $1,52(0,75)$ & $1,52(0,75)$ & $1,56(0,75)$ & $1,59(0,88)$ &, $735^{\star *}$ &, $505^{\star *}$ \\
\hline
\end{tabular}

\section{DISCUSIÓN}

Este estudio tuvo como objetivo desarrollar y validar una escala de figuras corporales para evaluar la percepción del tamaño corporal y la insatisfacción corporal en preescolares. Los resultados obtenidos con los pediatras expertos demostraron una alta correlación para la validez de constructo y una buena fiabilidad. El nivel de concordancia entre los expertos fue más alto con las figuras con sobrepeso y obesidad, por lo que el exceso de peso parece ser más claramente observable en las fotografías y figuras del instrumento.

Por otro lado, las correlaciones de Spearman con los niños demostraron una fiabilidad test-retest moderada-alta a partir de 5 años con ambas escalas, tal y como se hipotetizó, lo cual demuestra que las respuestas de los niños mayores son más consistentes en el tiempo, especialmente con las figuras percibidas, mientras que la fiabilidad fue algo menor para las figuras ideales. Este hecho 
podría deberse a que en estas edades los niños aún no tengan formado un ideal corporal, lo cual les lleva a seleccionar figuras diferentes en distintos días. En este sentido, se ha argumentado que a estas edades los niños aún no han desarrollado el pensamiento abstracto que les permite distinguir y comparar entre su yo real y lo que sería el yo ideal (Dunphy-Lelii, Hooley, McGivern, Guha y Skouteris, 2014; Papalia, Wendkos y Duskin, 2010). Dicho pensamiento abstracto comienza a emerger a partir de los 11 años y, gracias a él, los niños consiguen ir más allá de las experiencias concretas y desarrollar imágenes de circunstancias ideales. Un ejemplo de ello sería que pueden imaginar cómo sería un padre ideal y compararlo con el real. Por tanto, son capaces de pensar en el futuro y en lo que podrían ser (Santrock, 2007).

La escala diseñada por Collins (1991) para niños de mayor edad demostró ser fiable con índices similares a los obtenidos en este estudio. Por otro lado, Truby y Paxton (2008), con su escala de siete figuras corporales (Children's Body Image Scale -CBIS-) para niños de 7 a 11 años hallaron una fiabilidad testrestest superior a la del instrumento del presente estudio, tanto con la figura percibida como con la ideal.

Este resultado podría venir explicado por la madurez cognitiva de los participantes, cuyas ideas y respuestas son más estables en el tiempo. Truby y Paxton (2008), al igual que Lerner y Gellert (1969) demostraron que el género femenino era más consistente en sus respuestas, justificando que ello podría reflejar su superioridad y precocidad en el desarrollo cognitivo. Sin embargo, en el presente estudio, fueron los chicos quienes demostraron mayor fiabilidad, tanto en la elección de la figura percibida como la ideal, lo cual podría sugerir que los chicos de edades muy tempranas tienen más claras sus elecciones en relación a la imagen corporal. Esta claridad en sus respuestas podría justificar que sea el género masculino el que tiene una mayor exactitud en la percepción de su tamaño corporal (Ambrosi-Randic, 2000; Holub, 2008). Por el contrario, la menor consistencia en las respuestas de las chicas podría sugerir que éstas son más vulnerables y están más influidas por factores externos que les hacen modificar sus respuestas y tener una imagen corporal menos estable. En este sentido, Smolak (2004) afirma que las chicas son más presionadas socialmente que los chicos para alcanzar un determinado ideal corporal.

La presente investigación contribuye al campo de la imagen corporal mediante la aportación de un nuevo instrumento que, según nuestro conocimiento, es el primero válido y fiable para preescolares. Una fortaleza del PBS es que se compone de cuerpos y dimensiones antropométricas reales y no de dibujos subjetivos, lo que nos permite evaluar con precisión el componente perceptivo y cognifivo-afectivo de la imagen corporal. Además, la ausencia de rasgos faciales permite ser empleado con diferentes etnias. No obstante, al ser cuerpos reales, las imágenes no tienen exactamente la misma posición, las diferentes partes del cuerpo no están ubicadas a la misma distancia y los cambios de tamaño corporal no son consistentes. Otra fortaleza del estudio fue la participación de pediatras expertos con el objetivo de superar la limitación de validar el instrumento con niños. Finalmente, se destaca como fortaleza del instrumento el limitado rango de edad que incluye PBS, ya que como señalan Gardner y Brown (2010), los 
cuerpos de los niños están continuamente cambiando y es importante que se utilice una escala de figuras adecuada para su edad.

Como limitación del estudio cabe señalar el reducido número de participantes existente en algunos grupos de edad, ya que éstos están divididos por semestres (e.g., de 4 a 4,4 años) en lugar de por años completos de edad (e.g., existen 66 niños de 4 años).

Como prospectiva, sería valioso aplicar el instrumento para analizar la percepción del tamaño corporal y la insatisfacción corporal en preescolares, quienes han recibido menos atención en el estudio de imagen coporal. Para una adecuada comprensión del desarrollo y evolución de la imagen corporal desde edades tempranas, se requiere el uso de métodos mixtos que aporten tanto datos cuantitativos como cualitativos en relación con la percepción corporal y la insatisfacción corporal.

\section{CONCLUSIONES}

Los resultados muestran que PBS es un instrumento válido y fiable para preescolares, siendo recomendable utilizarlo a partir de 5 años. La fácil aplicación del instrumento permite ser utilizado por investigadores y docentes de Educación Infantil, con el fin de conocer la imagen corporal en las poblaciones más jóvenes y evitar problemas futuros de salud o trastornos relacionados con la imagen corporal como son la anorexia, bulimia o dismorfia muscular.

\section{REFERENCIAS BIBLIOGRÁFICAS}

Ambrosi-Randic, N. (2000). Perception of current and ideal body size in preschool age children. Perceptual and Motor Skills, 90(3), 885-889. https://doi.org/10.2466/pms.2000.90.3.885

Ambrosi-Randic, N. y Tokuda, K. (2004). Perceptions of body image among Japanese and Croatian children of preschool age. Perceptual and Motor Skills, 98(2), 473-478. https://doi.org/10.2466/pms.98.2.473-478

Barnsley, R. H., Thompson, A. H. y Barnsley, P. E. (1985). Hockey success and birthdate: The RAE. Canadian Association for Health, Physical Education, and Recreation, 51(8), 23-28.

Burgess, J. N. y Broome, M. E. (2012). Perceptions of weight and body image among preschool children: a pilot study. Pediatric Nursing, 38(3), 147-176. Recuperado de http://www.pediatricnursing.net/

Collins M. E. (1991). Body figure perceptions and preferences among preadolescent children. International Journal of Eating Disorders, 10(2), 199-208. https://doi.org/10.1002/1098-108X

Cramer, P. y Steinwert, T. (1998). Thin is good, fat is bad: How early does it begin? Journal of Applied Developmental Psychology, 19(3), 429-451. https://doi.org/10.1016/S0193-3973(99)80049-5

Damiano, S., Gregg, K., Spiel, E., McLean, S., Wertheim, E. y Paxton, S. (2015). Relationships between body size attitudes and body image of 4-year-old boys and girls, and attitudes of their fathers and mothers. Journal of Eating Disorders, 3(16), 1-10. https://doi.org/10.1186/s40337-015-0048-0 
Davison, K. K., Markey, C. N. y Birch, L. L. (2000). Etiology of body dissatisfaction and weight concerns among 5-year-old girls. Appetite, 35(2), 143-151. https://doi.org/10.1006/appe.2000.0349

Dohnt, H. y Tiggemann, M. (2006a). Body image concerns in young girls: the role of peers and media prior to adolescence. Journal of Youth and Adolescence, 35(2), 141-151. https://doi.org/10.1007/s10964-005-9020-7

Dohnt, H. y Tiggemann, M. (2006b). The contribution of peer and media influences to the development of body satisfaction and self-esteem in young girls: A prospective study. Developmental Psychology, 42(5), 929936. https://doi.org/10.1037/0012-1649.42.5.929

Dunphy-Lelii, S., Hooley, M., McGivern, L., Guha, A. y Skouteris, H. (2014). Preschoolers' body-knowledge inaccuracy: perceptual self-deficit and attitudinal bias. Early Child Development and Care, 184(11), 1757-1768. https://doi.org/10.1080/03004430.2014.881357

Fleiss, J. L. (1981). The measurement of interrater agreement, statistical methods for rates and proportions. New York: John Wiley \& Sons.

Gardner, R. M. (2012). Measurement of perceptual body image. En T. F. Cash (Ed.), Encyclopedia of body image and human appearance, volumen 2 (pp. 526-532). https://doi.org/10.1016/B978-0-12-384925-0.00083-3

Gardner, R. M. y Brown, D. L. (2010). Body image assessment: A review of figural drawing scales. Personality and Individual Differences, 48(2), 107-111. doi: 10.1016/j.paid.2009.08.017

Gardner, R. M., Jappe, L. M. y Gardner, L. (2009). Development and validation of a new figural drawing scale for body-image assessment: The BIAS-BD. Journal of Clinical Psychology, 65(1), 113-122. https://doi.org/10.1002/jclp.20526

Gómez-Mármol, A., Sánchez-Alcaraz, B. J., Molina-Saorín, J. y Bazaco, M. J. (2017). Violencia escolar y autoimagen en adolescents de la region de Murcia (España). Revista Internacional de Medicina y Ciencias de la Actividad Física y del Deporte, 17(68), 677-692. https://doi.org/10.15366/rimcafd2017.68.007

Hayes, S. y Tantleff-Dunn, S. (2010). Am I too fat to be a princess? Examining the effects of popular children's media on young girls' body image. British Journal of Developmental Psychology, 28(2), 413-426. https://doi.org/10.1348/026151009X424240

Hill, A. J. (2012). Measurement of body image in childhood. En T. F. Cash (Ed.), Encyclopedia of body image and human appearance, volume 2 (pp. 521525). London: Academic Press. https://doi.org/10.1016/B978-0-12384925-0.00082-1

Holub, S. C. (2008). Individual differences in the anti-fat attitudes of preschoolchildren: The importance of perceived body size. Body Image, 5(3), 317321. https://doi.org/10.1016/j.bodyim.2008.03.003

Landeta J. (2002). El método Delphi: una técnica de previsión del futuro. Barcelona: Ariel.

Landis, R. y Koch G. G. (1977). The measurement of observer agreement for categorical data. Biometrics, 33(1), 159-174. https://doi.org/10.2307/2529310

Lerner, R. y Gellert, E. (1969). Body build identification, preference, and aversion in children. Developmental Psychology, 1(5), 456-462. http://doi.org/10.1037/h0027966 
Li, Y., Hu, X., Ma, W., Wu, J. y Ma, G. (2005). Body image perceptions among Chinese children and adolescents. Body Image, 2(2), 91-103. https://doi.org/10.1016/j.bodyim.2005.04.001

Lombardo, C., Battagliese, G., Pezzuti, L. y Lucidi, F. (2014). Validity of a figure rating scale assessing body size perception in school-age children. Eating Weight Disorders, 19(3), 329-336. https://doi.org/1007/s40519-013-0085$\underline{0}$

Matas, A. (2018). Diseño del formato de escalas tipo Likert: un estado de la cuestión. Revista electronica de investigación educativa, 20(1), 38-47. https://doi.org/10.24320/redie.2018.20.1.1347

McCabe, M. P., Ricciardelli, L. A., Stanford, J., Holt, K., Keegan, S. y Miller, L. (2007). Where is all the pressure coming from? Messages from mothers and teachers about preschool children's appearance, diet and exercise. European Eating Disorders Review, 15(3), 221-230. http://doi.org/10.1002/erv.717

Meers, M., Koball, A., Wagner, M., Laurene, K. y Musher-Eizenman, D. (2011). Assessing anti-fat bias in preschoolers: A comparison of a computer generated line-drawn figure array and photographic figure array. Body Image, 8(3), 293-296. https://doi.org/10.1016/j.bodyim.2011.04.006

Musher-Eizenman, D., Holub, S., Edwards-Leeper, L., Persson, A. y Goldstein, S. (2003). The narrow range of acceptable body types of preschoolers and their mothers. Applied Developmental Psychology, 24(2), 259-272. https://doi.org/10.1016/S0193-3973(03)00047-9

Organización Mundial de la Salud (2006). The WHO child growth standards. Recuperado de http://www.who.int/childgrowth/en/

Organización Mundial de la Salud (2007). WHO AnthroPlus software V 1.0.4. Recuperado de http://www.who.int/growthref/tools/en/

Palacios, J., Marchesi, A. y Coll, C. (1990). Desarrollo psicológico y educación. Psicología evolutiva. Madrid: Alianza.

Pallan, M., Hiam, L., Duda, J. y Adab, P. (2011). Body image, body dissatisfaction and weight status in south asian children: a cross-sectional study. BMC Public Health, 11(21), 1-8. https://doi.org/10.1186/1471-2458-11-21

Papalia, D., Wendkos, S. y Duskin, R. (2010). Desarrollo humano. México: McGraw-Hill.

Paxton, S. J. y Damiano, S. R. (2017). The development of body image and weight bias in childhood. En J. B. Benson (Ed.), Advances in child development and behavior (volume 52) (pp. 269-298). San Diego: Elsevier.

Ra, J. S., Yun, H. J. y Cho, Y. H. (2016). Teachers' influence on weight perceptions in preschool children. Applied Nursing Research, 31, 111-116. https://doi.org/10.1016/j.apnr.2016.01.005

Rodgers, R. F., Paxton, S. J. y McLean, S. A. (2014). A biopsychosocial model of body image concerns and disordered eating in early adolescent girls. Journal of Youth and Adolescence, 43, 814-823. https://doi.org/10.1007/s10964-013-0013-7

Santrock, J. W. (2007). Desarrollo infantil. México: McGraw Hill.

Sociedad Internacional para el Avance de la Cineantropometría -ISAK- (2001). Normas internacionales para la valoración antropométrica. Australia: Librería Nacional. 
Smolak, L. (2004). Body image in children and adolescents: where do we go from here? Body Image, 1(1), 15-28. https://doi.org/10.1016/S17401445(03)00008-1

Smolak, L. (2012). Body image development in childhood. En T. F. Cash y L. Smolak (Eds.), Body image. A handbook of science, practice and prevention (pp. 67-75). New York: Guilford Publications.

Stunkard, A. J., Sorensen, T. y Schulsinger, F. (1983). Use of de Danish adoption register for the study of obesity and thinness. Association for Research in Nervous and Mental Disease, 60, 115-120. Recuperado de https://www.ncbi.nlm.nih.gov/pubmed/6823524

Swami, V., Salem, N., Furnham, A. y Tovée M. J. (2008). Initial examination of the validity and reliability of the female photographic figure rating scale for body image assessment. Personality and Individual Differences, 44, 17521761. https://doi.org/10.1016/j.paid.2008.02.002

Tiggemann, M. y Pennington, B. (1990). The development of gender differences in body size dissatisfaction. Australian Psychologist, 25(3), 306-313. https://doi.org/10.1080/00050069008260025

Thompson, J. K. (1990). Body image disturbance. Assessment and treatment. New York: Pergamon press.

Tremblay, L., Lovsin, T., Zecevic, C. y Larivière, M. (2011). Perceptions of self in 3-5 year-old children: A preliminary investigation into the early emergence of body dissatisfaction. Body Image, 8(3), 287-292. https://doi.org/10.1016/j.bodyim.2011.04.004

Truby, H. y Paxton, S. J. (2002). Development of the Children's Body Image Scale. British Journal of Clinical Psychology, 41(2), 185-203. https://doi.org/10.1348/014466502163967

Truby, H. y Paxton, S. J. (2008). The Children's Body Image Scale: Reliability and use with international standards for body mass index. British Journal of Clinical Psychology, 47(1), 119-124. https://doi.org/10.1348/014466507X251261

Wells, M., Goldstein, L. N. y Bentley, A. (2018). The use of body habitus reference images improves the ability of novices to accurately estimate children's weight using the pawper XL tape system. Journal of Emergency Medicine, 54(2), 165-175. https://doi.org/10.1016/j.jemermed.2017.10.009

Wong, Y., Chand Y. J. y Lin C. J. (2013). The influence of primary caregivers on body size and self-body image of preschool children in taiwan. Asia Pacific Journal of Clinical Nutrition, 22(2), 283-291. https://doi.org/10.6133/apjen.2013.22.2.05

$\mathrm{Xu}, \mathrm{T} . \mathrm{y}$ Nerren, J. S. (2016). Investigation young children's perceptions of body size and healthy habits. Early Childhood Education Journal, 45(4), 499507. https://doi.org/10.1007/s10643-016-0786-x

Número de citas totales / Total references: 50 (97.9\%) Número de citas propias de la revista / Journal's own references: 1 (2\%)

Rev.int.med.cienc.act.fís.deporte - vol. 21 - número 83 - ISSN: 1577-0354 